\title{
Innate and Adaptive Immune Responses to SARS-CoV-2 in Humans: Relevance to Acquired Immunity and Vaccine Responses
}

\author{
Stanley Jordan ${ }^{1}$ \\ ${ }^{1}$ Cedars Sinai Med Ctr
}

December 1, 2020

\begin{abstract}
The factors responsible for the spectrum of COVID-19 disease severity and the genesis and nature of protective immunity against COVID-19 remain elusive. Multiple studies have investigated the immune responses to COVID 19 in various populations, including those without evidence of COVID 19 infection. Information regarding innate and adaptive immune responses to the novel SARS-CoV-2 has evolved rapidly. Here, data are accumulating defining disease phenotypes that aid in rational and informed development of new therapeutic approaches for the treatment of patients infected with SARS-CoV-2 and the development of novel vaccines. In this article, we summarize data on important innate immune responses including cytokines, specifically IL-6 and complement, and explore potential treatments. We also examine adaptive immune responses and derivative therapeutics such as monoclonal antibodies directed at spike proteins. Finally, we explore data on real-time assessments of adaptive immune responses which include CD4+/CD8+ T-cells, NKT-cells, memory B-cells, and T-follicular cells with specificities for COVID-19 peptides in infected individuals and normals. Data of two novel vaccines have been released, both showing $>95 \%$ efficacy in preventing SARS-CoV-2 infection. Analysis of humoral and cellular responses to the vaccines will determine the robustness and durability of protection. In addition, long-term assessment of SARS-CoV-2 memory B and T-cell mediated immune responses in patients recovering from an infection or those with cross-reactive immunologic memory will help to define risk for future SARS-CoV infections. Finally, patients recovering from SARS-CoV-2 infection may experience prolonged immune activation likely due to T-cell exhaustion. This will be an important new frontier for study.
\end{abstract}

\section{Hosted file}

Innate \& Adaptive Immunity to COVID19-1 FINAL.pdf available at https://authorea.com/users/ 380273/articles/496327-innate-and-adaptive-immune-responses-to-sars-cov-2-in-humansrelevance-to-acquired-immunity-and-vaccine-responses 